\title{
Dicyanovinyl-quinquethiophenes with varying alkyl chain lengths: Investigation of their performance in organic devices
}

\author{
Kerstin Schulze, ${ }^{1, a)}$ Moritz Riede, ${ }^{1}$ Eduard Brier, ${ }^{2}$ Egon Reinold, ${ }^{2}$ Peter Bäuerle, ${ }^{2}$ and \\ Karl Leo ${ }^{1, b)}$ \\ ${ }_{1}^{1}$ Institut für Angewandte Photophysik, Technische Universität Dresden, George-Bähr-Straße 1, \\ 01069 Dresden, Germany \\ ${ }^{2}$ Institut für Organische Chemie II und Neue Materialien, Universität Ulm, 89081 Ulm, Germany
}

(Received 3 June 2008; accepted 13 August 2008; published online 13 October 2008)

\begin{abstract}
We compare between two derivatives of dicyanovinyl-quinquethiophenes with different alkyl side chain lengths. Both materials show comparable open circuit voltages $V_{\text {oc }}$ in organic solar cells with fullerene $C_{60}$ as acceptor, as expected since they have the same highest occupied molecular orbital energy. However, differences in the current-voltage-characteristics, particularly in the fill factor, are observed. We analyze both derivatives in hole-only devices and find a difference in the hole injection between the doped hole transport layer and the oligothiophenes. Additionally, we determine the hole mobility of the two materials and explain the different behaviors of the two materials in solar cells. (C) 2008 American Institute of Physics. [DOI: 10.1063/1.2990071]
\end{abstract}

\section{INTRODUCTION}

In comparison to inorganic photovoltaics, organic solar cells have the potential to achieve lower cost: organic materials usually exhibit high absorption coefficients, such that thin layers of several tenths of nanometers of the comparatively cheap and abundant organic compounds are sufficient for photovoltaic devices. Additionally, the possibility of rollto-roll deposition at room temperature reduces cost. Both small molecule or polymer organic solar cells have already achieved power conversion efficiencies of up to $5-6 \% .^{1-3}$ Still, organic solar cells have to be improved further, especially concerning efficiency and device stability.

One possibility of improving efficiency is the use of optimized electron donor and acceptor materials to minimize energetic losses during exciton separation. We use here quinquethiophenes with a low lying highest occupied molecular orbital (HOMO) [approximately $-5.6 \mathrm{eV}$ determined with ultraviolet photoelectron spectroscopy (UPS)] instead of the frequently used metal-phthalocyanines [e.g., HOMO of zinc phthalocyanine: $\left.-5.2 \mathrm{eV}^{4}\right]$ as donor when using fullerene $C_{60}$ as acceptor. We have shown before that derivatives of this material class are promising donors in combination with $C_{60}$ in small molecule organic solar cells. ${ }^{5,6}$ These solar cells reach an open circuit voltage $V_{\text {oc }}$ of approximately $1 \mathrm{~V}$ and power efficiencies of up to $3.4 \%$. The approximately $0.4 \mathrm{~V}$ higher $V_{\text {oc }}$ compared to phthalocyanine based solar cells (in planar heterojunction solar cells) is caused by the increase in the ionization potential of the oligothiophene compared to the phthalocyanine.

In our small molecule solar cells, the photoactive materials are usually embedded between a hole transport layer (HTL) and a combination of a thin 4,7-diphenyl-1,10phenanthroline (BPhen) layer, with aluminum serving as

\footnotetext{
a)Electronic mail: schulze@iapp.de.

b) Author to whom correspondence should be addressed. Electronic mail: leo@iapp.de. FAX:++49-351-46337065.
}

cathode. Because of the high ionization potential of the oligothiophene, the contact to the HTL has a major influence on the $I V$-curve of the device. ${ }^{6}$ When using HTLs with high ionization potential, the difference between the built-in potential $V_{\text {bi }}$ and the open circuit voltage $V_{\text {oc }}$ leads to a $S$-shape in the $I V$-curve. The $V_{\mathrm{bi}}$ is defined by the difference of the Fermi levels at the contacts, in our case in the doped HTL and the BPhen/Al-contact. The $V_{\mathrm{oc}}$ is defined by the difference of the quasi-Fermi levels in the donor and acceptor under illumination. It was shown that this problem can be reduced or avoided by using an HTL with nearly identical HOMO values compared to the oligothiophene. ${ }^{5,7}$ As we will show in this paper, the $S$-shape does not only depend on the energetic difference between the HOMO-levels of the HTL and the oligothiophenes but additionally on the type of the oligothiophene.

We analyze the interface between HTLs and two oligothiophene derivatives in solar cells and hole-only devices. The chemical structures of the two dicyanovinylquinquethiophene (DCV5T) derivatives DCV5T-Bu and DCV5T-Et are shown in Fig. 1(a). These molecules consist of five thiophene units and dicyanovinyl-groups as endgroups as well as two alkyl side chains at the second and

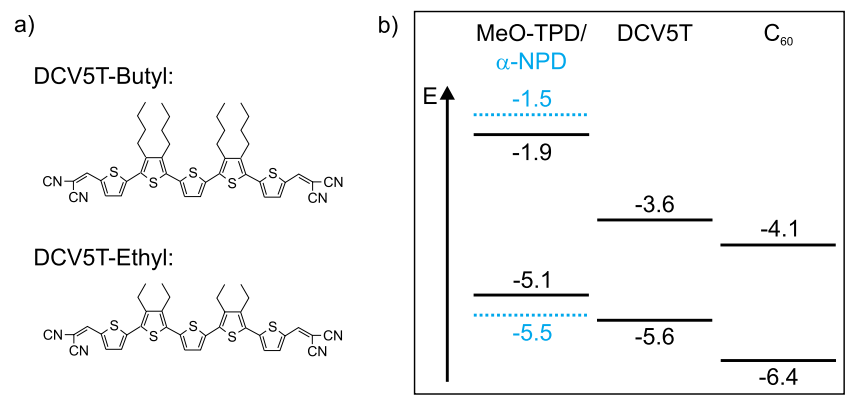

FIG. 1. (Color online) (a) Chemical structure of the DCV5T with butyl- or ethyl-sidechains (DCV5T-Bu and DCV5T-Et). (b) Energetic alignment of HOMO and LUMO of the donor and acceptor as well as the adjacent hole transport material MeO-TPD and $\alpha$-NPD of the four devices. 
TABLE I. Layer sequence of the HTL and donor in the four solar cells: ITO/1 nm Au/HTL/donor/52 nm $C_{60} / 6 \mathrm{~nm}$ BPhen/100 nm Al.

\begin{tabular}{llc}
\hline \hline Device & \multicolumn{1}{c}{$\mathrm{HTL}$} & Donor \\
\hline $\mathrm{A}$ & $40 \mathrm{~nm} p$-doped MeO-TPD/5 nm MeO-TPD & $9.8 \mathrm{~nm}$ DCV5T-Bu \\
B & $30 \mathrm{~nm} p$-doped TNATA $/ 10 \mathrm{~nm}$ & $9.8 \mathrm{~nm}$ DCV5T-Bu \\
& $p$-doped $\alpha$-NPD $/ 5 \mathrm{~nm} \alpha$-NPD & \\
$\mathrm{C}$ & $40 \mathrm{~nm} p$-doped MeO-TPD $/ 5 \mathrm{~nm}$ MeO-TPD & $10 \mathrm{~nm}$ DCV5T-Et \\
$\mathrm{D}$ & $10 \mathrm{~nm} p$-doped $\alpha$-NPD $/ 5 \mathrm{~nm} \alpha$-NPD & $10 \mathrm{~nm}$ DCV5T-Et \\
\hline \hline
\end{tabular}

fourth thiophene. The only structural difference between DCV5T-Bu and DCV5T-Et is the length of the alkyl chains, which varies between $\mathrm{C}_{4} \mathrm{H}_{9}(\mathrm{Bu})$ and $\mathrm{C}_{2} \mathrm{H}_{5}(\mathrm{Et})$.

\section{EXPERIMENTAL}

For the analysis of the behavior of these derivatives in organic solar cells, two different hole transport materials $\mathrm{N}, \mathrm{N}, \mathrm{N}^{\prime}, \mathrm{N}^{\prime}$-tetrakis(4-methoxyphenyl)-benzidine (MeO-TPD) (Sensient, Wolfen) and $\mathrm{N}, \mathrm{N}^{\prime}-$ di(naphthalen-1-yl)-N, $\mathrm{N}^{\prime}$-diphenyl-benzidine $\quad(\alpha$-NPD) (Sensient, Wolfen) are used. These materials are $p$-doped using the proprietary acceptor dopant NDP-2 from Novaled AG, Dresden (doping ratio of approximately $1.3 \mathrm{wt} \%$ in MeO-TPD and $10 \mathrm{wt} \%$ in $\alpha$-NPD). Between the $p$-doped HTL and the oligothiophene, $5 \mathrm{~nm}$ of the intrinsic HTL is deposited to avoid exciton quenching, which occurs by a direct contact between the dopant molecules and the photoactive materials, especially at a contact to a highly doped HTL. ${ }^{8}$ In the hole-only devices, we use additionally a p-doped zinc phthalocyanine ( $\mathrm{ZnPc}$ ) (Alfa Aesar $\mathrm{GmbH} \&$ Co. KG, Karlsruhe) layer in direct contact to Au to provide an ohmic contact. The $p$-doping ratio is set to approximately $2 \mathrm{wt} \%$. The materials are cleaned at least twice by thermal gradient sublimation before evaporation, except the oligothiophenes and the acceptor dopant, which are used as received.

All solar cells use the following layer sequence: indium tin oxide (ITO) $/ 1 \mathrm{~nm}$ Au/HTL/donor $/ 52 \mathrm{~nm} \quad C_{60} / 6 \mathrm{~nm}$ $\mathrm{BPhen} / 100 \mathrm{~nm}$ Al. The materials and layer thicknesses of the HTL and donor are summarized in Table I. A scheme of the energetic alignment of the HOMO and lowest unoccupied molecular orbital (LUMO) of the acceptor, donor, and adjacent HTLs is shown in Fig. 1(b). The additional p-doped 4, 4' , 4"-tris(2-naphthylphenylamino)-triphenylamin (TNATA) layer in device B does not influence the $I V$-curve, but lowers the possibility of shortcuts when using relatively rough ITO substrates. The structure of the hole-only devices is: ITO/ $1 \mathrm{~nm} \quad \mathrm{Au} / 30 \mathrm{~nm} \quad p$-doped $\alpha$-NPD/ $x \mathrm{~nm}$ oligothiophene/20 nm $p$-doped $\alpha$-NPD/11.4 nm $p$-doped $\mathrm{ZnPc} / 50 \mathrm{~nm}$ Au using either DCV5T-Bu or DCV5T-Et with different thicknesses (DCV5T-Bu: 45, 60, and $75 \mathrm{~nm}$; DCV5T-Et: 75, 90, and $150 \mathrm{~nm}$ ).

The devices with a photoactive area of $4-7 \mathrm{~mm}^{2}$ are deposited in a UHV multichamber system (typical pressure of $10^{-8}-10^{-7}$ mbar during evaporation). Current voltage measurements are carried out in a nitrogen glovebox using a Source Measurement Unit 236 (Keithley) and illumination is provided by a sun simulator (Hoenle AG). Intensity calibra-
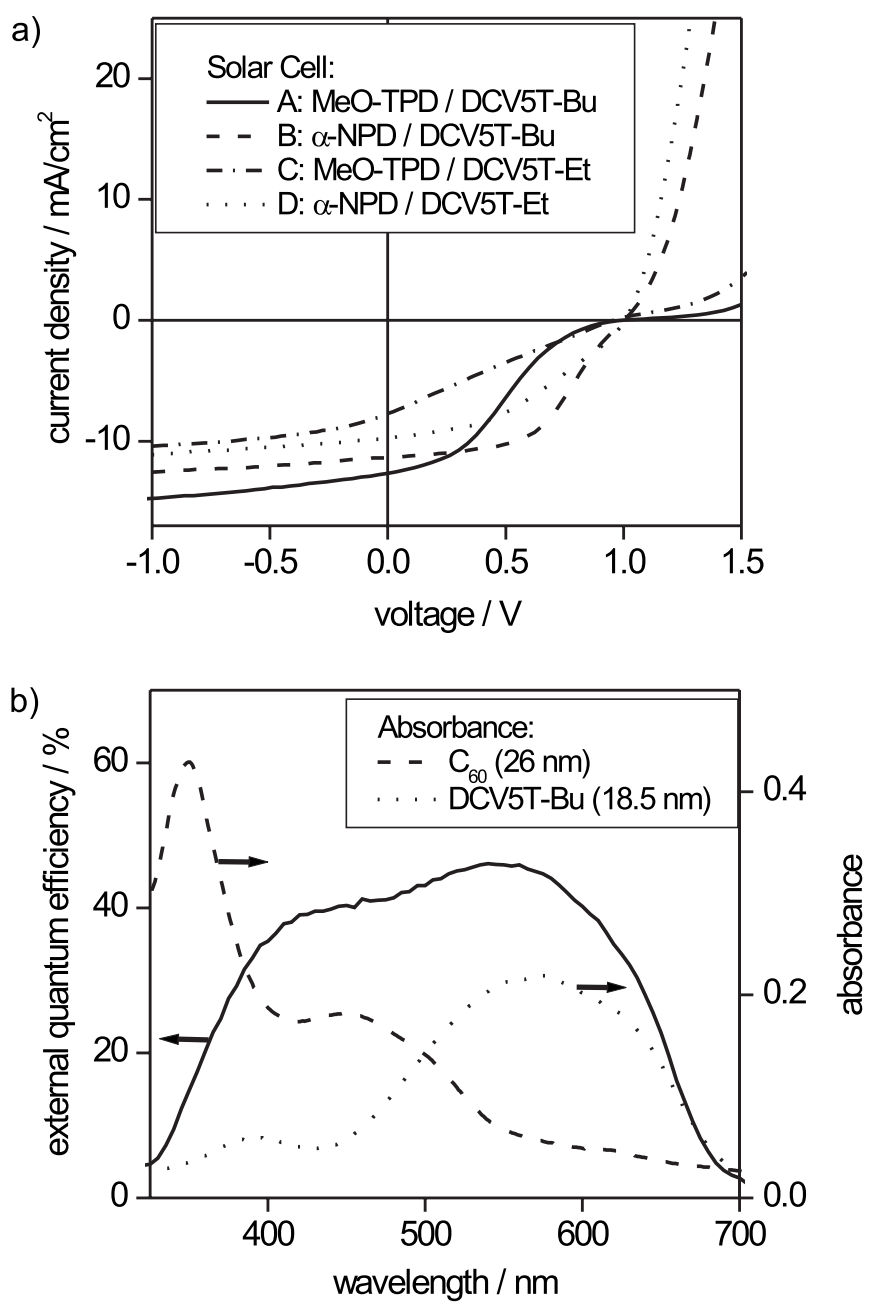

FIG. 2. (a) $I V$-curves of the devices using either DCV5T-Bu or DCV5T-Et as donor and MeO-TPD or $\alpha$-NPD as contacting HTL. (b) Absorption of thin films of the materials $C_{60}$ (dashed line) and DCV5T-Butyl (dotted line) and spectrum of the external quantum efficiency of device B (straight line).

tion of the sun simulator is done by using an outdoor reference cell calibrated by the Fraunhofer Institute for Solar Energy Systems (Freiburg, Germany). The external quantum efficiency (EQE) measurement is done by using a homemade setup based on a xenon lamp and a grating monochromator. The setup is calibrated using a Newport powermeter. Absorption and photoluminescence spectra are recorded with standard spectrometers from Shimadzu and SPEX, respectively.

\section{RESULTS AND DISCUSSION}

The $I V$-curves of the devices A-D are presented in Fig. 2(a). The $I V$-curve of device B has already been shown in Ref. 9 and the $I V$-curve of device $\mathrm{D}$ has been presented in Ref. 6 but both solar cells were discussed in another context. Additionally, the absorbance of the photoactive materials $C_{60}$ and DCV5T-Bu in thin film and the external quantum efficiency spectrum of device B are shown in Fig. 2(b). As the current density of this device scales linearly with light intensity, we integrate this spectrum with the AM 1.5 spectrum to estimate the spectral mismatch for this cell. For the other cells, we assume a comparable mismatch factor as that determined for device B. The characteristic parameters of the 
TABLE II. Short circuit current density $j_{\mathrm{sc}}$ (normalized at a sun intensity of the sun simulator of $100 \mathrm{~mW} / \mathrm{cm}^{2}$ determined with the reference cell without spectral mismatch), open circuit voltage $V_{\mathrm{oc}}$, fill factor (FF), saturation factor, and estimated efficiency $\eta$ (spectral mismatch of 1.34 as it was estimated for device B is taken into account) of devices A, B, C, and D.

\begin{tabular}{lccccccc}
\hline \hline Device & Donor & HTL & $\begin{array}{c}j_{\text {sc }} \\
{\left[\mathrm{mA} / \mathrm{cm}^{2}\right]}\end{array}$ & $\begin{array}{c}V_{\text {oc }} \\
{[\mathbf{V}]}\end{array}$ & $\begin{array}{c}\text { FF } \\
{[\%]}\end{array}$ & $\begin{array}{c}\text { Saturation } \\
j_{-1 \mathrm{v}} / j_{\mathrm{sc}}\end{array}$ & $\begin{array}{c}\text { Estimate } \eta \\
{[\%]}\end{array}$ \\
\hline A & DCV5T-Bu & MeO-TPD & 9.7 & 1.00 & 28.2 & 1.16 & 2.0 \\
$\mathrm{~B}$ & DCV5T-Bu & $\alpha$-NPD & 8.9 & 1.00 & 50.5 & 1.10 & 3.4 \\
$\mathrm{C}$ & DCV5T-Et & MeO-TPD & 7.0 & 0.95 & 25.0 & 1.42 & 1.2 \\
D & DCV5T-Et & $\alpha$-NPD & 8.4 & 1.00 & 39.9 & 1.15 & 2.5 \\
\hline \hline
\end{tabular}

photovoltaic cells are given in Table II. The current densities are the values determined under the sun simulator, whereas for the estimated efficiencies, the spectral mismatch factor was taken into account.

All devices reach a comparable open circuit voltages $V_{\mathrm{oc}}$ of approximately $1 \mathrm{~V}$. This is due to the $V_{\text {oc }}$ being determined by the difference of the quasi-Fermi levels in the donor and acceptor under illumination, which should be independent of the HOMO-value of the hole transport material. A comparable open circuit voltage of the devices means that the ionization potential of both derivatives has to be nearly identical. By using UPS we determine the ionization potential of approximately $5.6 \mathrm{eV}$ for both derivatives, which means that the ionization potential is independent of the length of the alkyl chains of these quinquethiophenes. However, a significant difference in the performance depending on the HTL is visible, analogous to our previous results. ${ }^{5}$ Caused by the low lying HOMO of the oligothiophene $(-5.6 \mathrm{eV})$, the difference between $V_{\mathrm{oc}}$ and $V_{\mathrm{bi}}$ when using MeO-TPD (HOMO: $-5.1 \mathrm{eV}$, determined with UPS) as HTL is much higher compared to the devices with $\alpha$-NPD (HOMO: $-5.5 \mathrm{eV}$, determined with UPS). In the voltage range between $V_{\mathrm{bi}}$ and $V_{\text {oc }}$, the free charge carriers are only driven by diffusion to the contacts. This causes a higher recombination rate, leading to a $S$-shape in the $I V$-curve.

However, the two oligothiophene derivatives exhibit a difference in the shape of the $I V$-curves even for the same HTL. This is especially obvious in solar cells B and D, both using $\alpha$-NPD as adjacent hole transport material. UPS measurements indicate that these differences are not caused by different energetic alignments; the data do not yield a difference in energy level alignment between the HTL, the oligothiophenes, and the fullerene. We determine the formation of an almost identical injection barrier between a $p$-doped $\alpha$-NPD-layer and the oligothiophene derivatives of approximately $0.22-0.25 \mathrm{eV}$. It seems that the $S$-shape in the device using DCV5T-Bu is still pronounced but weaker in the device with DCV5T-Et. This means that effects beyond the energetic difference between the HOMO of the donor and the HTL must be responsible for the shape of the $I V$-curve. We have observed such behavior for many devices. In the following, we give a possible explanation for the differences in the solar cell performance depending on the oligothiophene derivative.

To further study these effects, we have prepared holeonly devices to analyze both the hole injection behavior between $p$-doped $\alpha$-NPD and the oligothiophenes and the hole mobilities on DCV5T-Bu and DCV5T-Et. Two structures have been prepared, each having the following layer sequence: ITO/1 nm Au/30 nm $p$-doped $\alpha$-NPD/75 nm oligothiophene/20 nm $p$-doped $\alpha$-NPD/11.4 nm $p$-doped $\mathrm{ZnPc} / 50 \mathrm{~nm}$ Au using either DCV5T-Bu or DCV5T-Et. The $I V$-curves of these devices are shown in Fig. 3(a). The hole injection via ITO is given for negative applied voltages and via the Au contact for positive applied voltages.

Two significant points are visible. First, the hole-only device using DCV5T-Et reaches higher current densities at the same applied voltages compared to the device with DCV5T-Bu. Second, for the device using DCV5T-Et, a symmetrical $I V$-curve is observed, whereas the cell using DCV5T-Bu shows an asymmetrical curve. For a better visualization, the absolute value of the ratio between forward current and backward current is shown in Fig. 3(b). This indicates a better hole injection for $p$-doped $\alpha$-NPD and DCV5T-Et via the ITO/ $p$-doped $\alpha$-NPD-contact than for DCV5T-Bu. The different behavior of the hole-only devices seems to depend on the length of the alkyl chain. Interaction of the molecules is possible between the cyanogroups and the hydrogenatoms $(\mathrm{CN} \cdots \mathrm{H})$ (Ref. 10) of the oligothiophene and $\alpha$-NPD molecules. The asymmetry of the device using DCV5T-Bu shows that the problem in hole injection is more pronounced when DCV5T-Bu is deposited on top of the $p$-doped $\alpha$-NPD layer, compared to a deposition of $p$-doped $\alpha$-NPD on DCV5T-Bu. An amorphous growth of DCV5T$\mathrm{Bu}$, as it was observed using atomic force microscopy, ${ }^{7}$ might be responsible for the better hole injection on the second interface (according to the deposition sequence), whereas the influence of the length of the alkyl chains is responsible for the problems in hole injection on the first interface to $p$-doped $\alpha$-NPD. An evaluation of the HOMO value of the oligothiophene and the $\alpha$-NPD reveals that the first interface has an energetic barrier and thus seems responsible for the injection problem. This difference is not observed in the device using DCV5T-Et due to the shorter alkyl chains, which might lead to a shorter distance between neighboring molecules. These findings might explain the $S$-shape of device B compared to device D, in which we do not observe such an effect. Problems in hole injection between the donor and the HTL lead in this case to a higher concentration of holes in the oligothiophene, which results in a higher recombination rate especially when the charge carriers are mainly driven to the contacts by diffusion (which is the case when voltages are applied between $V_{\mathrm{bi}}$ and $\left.V_{\mathrm{oc}}\right)$.

To further elaborate the differences in the 
a)

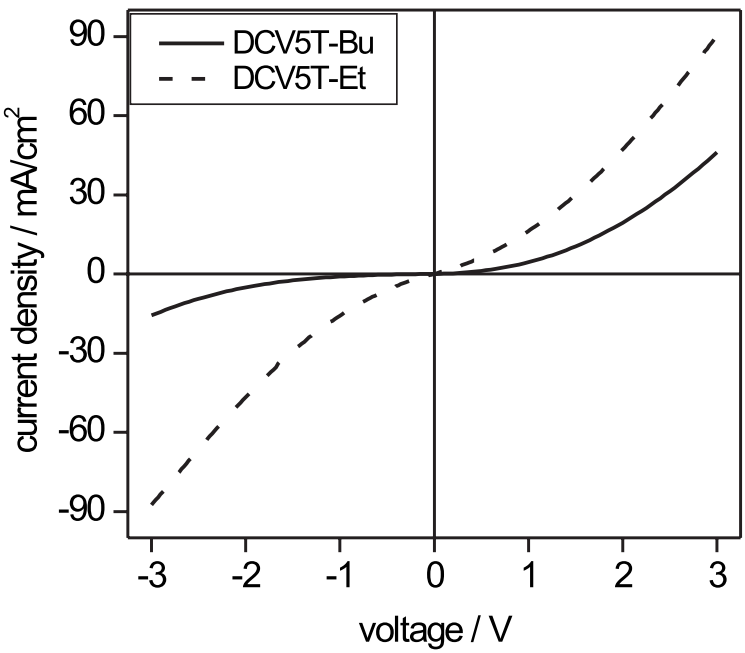

b)

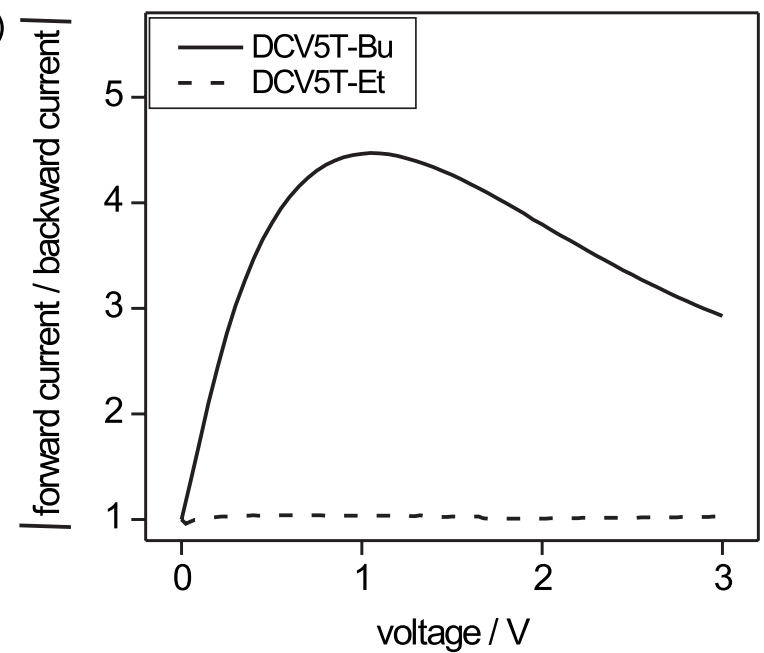

FIG. 3. (a) $I V$-curves of the devices using the following layer sequence: ITO/ $1 \mathrm{~nm} \quad \mathrm{Au} / 30 \mathrm{~nm} \quad p$-doped $\alpha$-NPD/75 nm oligothiophene $/ 20 \mathrm{~nm}$ $p$-doped $\alpha$-NPD/11,4 nm $p$-doped $\mathrm{ZnPc} / 50 \mathrm{~nm} \mathrm{Au}$. Using DCV5T-Et the $I V$-curve shows a symmetrical behavior, while using DCV5T-Bu an asymmetrical curve is observed. This is quantified in (b), where the absolute ratio between forward to backward current is shown.

$I V$-characteristics, we estimate the hole mobility $\mu$ in the oligothiophenes. We use the following equation for spacecharge limited currents ${ }^{11}$

$$
j=\frac{9}{8} \epsilon_{0} \epsilon_{r} \mu \frac{U^{2}}{d^{3}},
$$

where $d$ is the thickness of the intrinsic layer. The voltage drop in the $p$-doped layers is thereby neglected. To determine the hole mobility, we use the hole-only devices having the following structure: ITO/1 nm Au/30 nm $p$-doped $\alpha$-NPD/ $x \quad \mathrm{~nm} \quad$ oligothiophene/20 $\mathrm{nm} \quad p$-doped $\alpha$-NPD/11.4 nm $p$-doped ZnPc/50 nm Au. We choose 45, 60 , and $75 \mathrm{~nm}$ thick DCV5T-Bu layers and 75, 90, and 150 $\mathrm{nm}$ thick DCV5T-Et layers. Because of the higher hole currents, thicker layers for the ethyl-type derivative are necessary. The $I V$-curves are all shown in Fig. 4. When fitting using the relation $j \propto d^{a}$, we observe in both cases a value for $a$ somewhat higher than the expected -3 . Therefore, we assume that the current is not fully space charge limited and injection limitation occurs additionally. We use the relation a)

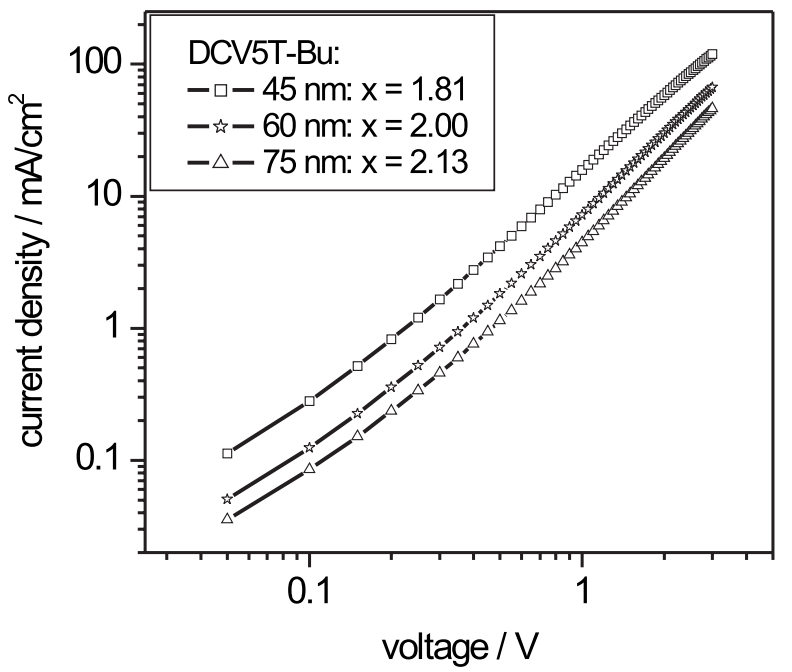

b)

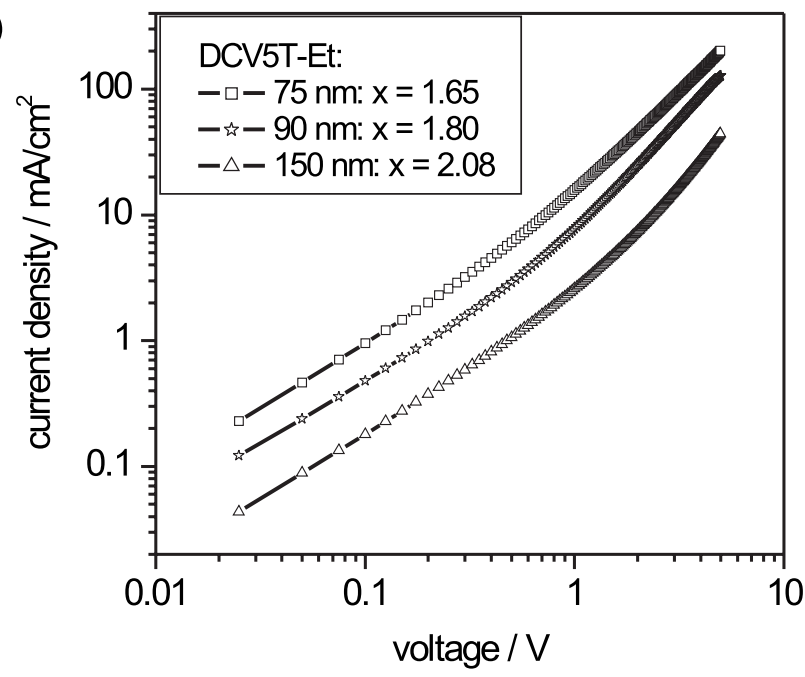

FIG. 4. IV-curves of hole-only devices using (a) 45, 60, and $75 \mathrm{~nm}$ DCV5T-Bu or (b) 75, 90, and $150 \mathrm{~nm}$ DCV5T-Et. In the inset, the parameter $x$ according to the relation $j \propto U^{x}$ is noted [used range: (a) $0.25-3 \mathrm{~V}$ and (b) $2.5-5 \mathrm{~V}]$.

$j \propto U^{x}$ for the curves in Fig. 4 to prove the behavior of $j \propto f(U)$. For the devices using DCV5T-Bu, we fit between 0.25 and $3 \mathrm{~V}$ and for the devices using DCV5T-Et, a good fit was found for applied voltages between 2.5 and $5 \mathrm{~V}$. We find $x \approx 2$ as expected from Eq. (1). By neglecting the injection limitation and using an average value of 3.5 for $\epsilon_{r}$, we estimate the hole mobility to $4.6 \times 10^{-6} \mathrm{~cm}^{2} / \mathrm{Vs}$ for DCV5T-Bu (extracted from the device using $60 \mathrm{~nm}$ DCV5T-Butyl) and $1.5 \times 10^{-5} \mathrm{~cm}^{2} / \mathrm{Vs}$ for DCV5T-Et (for the device using 150 $\mathrm{nm}$ DCV5T-Et). The hole mobility in DCV5T-Et is roughly one order of magnitude higher compared to DCV5T-Bu. The shorter alkyl chains in DCV5T-Et might lead to a lower distance between neighboring molecules, supporting the hopping transport to achieve higher hole mobility. Additionally, due to the $\mathrm{CN} \cdots \mathrm{H}$-interaction between the oligothiophene molecules mentioned above, interactions between alkyl chains are possible, which is more pronounced for longer chains. ${ }^{12}$ Unfortunately, we are not able to determine the distance between the molecules, because the molecules tend to grow amorphous and measurements using transmission electron microscopy do not show any crystallinity. 
Besides the difference of the hole injection, the difference in charge carrier mobility could be a possible explanation for the differences in the $I V$-curves of the solar cells B and $\mathrm{D}$. This applies especially at voltages around $V_{\mathrm{oc}}$, where the free charge carriers are mainly driven by diffusion and not supported by the internal electrical field. This leads to a higher concentration of free charge carriers in the oligothiophene layer and this high concentration especially at the interface between donor and acceptor leads to a higher recombination. The diffusion coefficient is directly connected with the mobility of the charge carriers and this means the lower the charge carrier mobilities, the higher is the recombination rate at the interface in our devices.

\section{CONCLUSION}

In conclusion, we present organic solar cells using DCV5T derivatives with two different alkyl chain lengths, DCV5T-Bu and DCV5T-Et, as electron donor in planar heterojunction devices. Both materials have an identical HOMO energy, but show a different behavior of the $I V$-curves. Possible explanations for the differences in the performance of the solar cells depending on the used oligothiophene derivatives are a difference in the hole injection between the HTL and the oligothiophene as well as a difference in hole mobility of the two materials. This can be explained by the difference in the distance between neighboring molecules, which influences the packing. Therefore the molecule with shorter sidechains, DCV5T-Et, exhibits less problems in hole injection between the HTL and oligothiophene and a higher hole mobility. This caused a lower free charge carrier density in DCV5T-Et, leading to lower recombination rates at applied voltages around $V_{\mathrm{oc}}$, so that an $S$-shape of the $I V$-curve does not appear.

\section{ACKNOWLEDGMENTS}

We thank Selina Olthof for the UPS measurements and the Deutsche Forschungsgemeinschaft via the Leibniz-Preis and the Fonds der chemischen Industrie for financial support.

${ }^{1}$ J. Xue, S. Uchida, B. P. Rand, and S. R. Forrest, Appl. Phys. Lett. 85, 5757 (2004)

${ }^{2}$ W. L. Ma, C. Y. Yang, X. Gong, K. Lee, and A. J. Heeger, Adv. Funct. Mater. 15, 1617 (2005).

${ }^{3}$ G. Li, V. Shrotriya, J. Huang, Y. Yao, T. Moriarty, K. Emery, and Y. Yang, Nature Mater. 4, 864 (2005).

${ }^{4}$ F. T. Reis, D. Mencaraglia, S. Oould Saad, I. Séguy, M. Oukachmih, P. Jolinat, and P. Destruel, Synth. Met. 138, 33 (2003).

${ }^{5}$ K. Schulze, C. Uhrich, R. Schueppel, K. Leo, M. Pfeiffer, E. Brier, E. Reinold, and P. Bäuerle, Adv. Mater. (Weinheim, Ger.) 18, 2872 (2006).

${ }^{6}$ K. Schulze, B. Maennig, K. Leo, Y. Tomita, C. May, J. Hüpkes, E. Brier, E. Reinold, and P. Bäuerle, Appl. Phys. Lett. 91, 073521 (2007).

${ }^{7}$ K. Schulze, Ph.D. thesis, Technische Universität Dresden, 2008.

${ }^{8}$ K. Schulze, C. Uhrich, R. Schüppel, K. Leo, M. Pfeiffer, E. Brier, E. Reinold, and P. Bäuerle, Proc. SPIE 6192, 61920C (2006).

${ }^{9}$ R. Schueppel, K. Schmidt, C. Uhrich, K. Schulze, D. Wynands, J. L. Brédas, E. Brier, E. Reinold, H. Bu, P. Baeuerle, B. Maennig, M. Pfeiffer, and K. Leo, Phys. Rev. B 77, 085311 (2008).

${ }^{10}$ J. Casado, T. M. Pappenfus, K. R. Mann, E. Orti, P. M. Viruela, B. Milian, V. Hernandez, and J. T. L. Navarrete, ChemPhysChem 5, 529 (2004).

${ }^{11}$ P. Mark and W. J. Helfrich, J. Appl. Phys. 33, 205 (1962).

${ }^{12}$ R. Azumi, G. Götz, T. Debaerdemaeker, and P. Bäuerle, Chem.-Eur. J. 6, 735 (2000). 